\title{
Arrow on domain conditions: a fruitful road to travel
}

\author{
Salvador Barberà*, Dolors Berga ${ }^{\dagger}$, and Bernardo Moreno \\ Final Version \\ June 12, 2019
}

\begin{abstract}
We stress the importance that Arrow attached to studying the role of domain conditions in determining the validity of his impossibility theorem, a subject to which he devoted two chapters of Social Choice and Individual Values. Then we partially survey recent results about the role of domain conditions on the possibility to design satisfactory aggregation rules and social choice functions, as a proof of the continued vitality of this subject, that he pioneered, as he did with so many others.
\end{abstract}

Keywords: Social welfare functions, social choice functions, restricted domains, single peakedness, selfishness, Arrow's Impossibiltity Theorem, dictatorship, connectedness.

*MOVE, Universitat Autònoma de Barcelona, and Barcelona GSE, Departament d'Economia i d'Història Econòmica, Edifici B, 08193 Bellaterra, Spain. E-mail: salvador.barbera@uab.cat

†Departament d'Economia, C/ Universitat de Girona 10, Universitat de Girona, 17003 Girona, Spain. E-mail: dolors.berga@udg.edu

†DDepartamento de Teoría e Historia Económica, Universidad de Málaga, Campus El Ejido, 29071 Málaga, Spain. E-mail: bernardo@uma.es 


\section{Introduction}

It is well understood that collective decision-making mechanisms can only satisfy interesting lists of desiderata if their domain of definition is somewhat restricted. In some cases, by limiting the scope of a theory to mechanisms that operate on a given class of domains, it becomes possible to identify, or even to fully characterize families of satisfactory mechanisms. In other cases, impossibilities still persist, in spite of such limitations, and this is equally relevant, especially when the domains appropriately represent social environments of interest.

There is an extensive literature on the subject, whose antecedents can be traced back to old times (see Gaertner, 2005), but the modern literature on the subject was sparkled by the fundamental works of Duncan Black (see Black, 1948 and 1958) and Kenneth Arrow (see Arrow, 1951-1963). The purpose of this essay is to comment on the nature and the role of restricted domains in the analysis of a variety of economic and political situations. More specifically, we will show how research on this topic is still active and fruitful, proving once again the far reaching influence of Arrow's work in so many directions. Gaertner (2001, 2002) has provided insightful overviews of work on domain restrictions up to the beginning of this century. In addition, specific approaches and subjects where domain restrictions play a crucial role are the object of several extensive surveys in the Handbook of Social Choice and Welfare (see Arrow, Sen, and Suzumura, 2002 and 2010). We shall refer to some of the important results already discussed in these works, but mostly insist on recent contributions that witness the continued fertility of the field.

\section{Arrow on domain conditions}

In Chapter V of Social Choice and Individual Values, after proving his General Possibility Theorem, Arrow restated this fundamental result in the following terms: "If we exclude the possibility of interpersonal comparisons of utility, then the only methods of passing from individual tastes to social preferences which will be satisfactory and which will be defined for a wide range of sets of individual orderings are either imposed or dictatorial". Then he concluded the chapter by announcing that the rest of the book (Chapters VI and VII, if we exclude the Notes in Chapter VIII) would be devoted to examine the meaning of the condition that "the method of forming a social ordering should work properly for a wide range of sets of individual orderings". In these late chapters, Arrow discussed three important instances in which relevant domains would need to be significantly restricted and the relevance of his impossibility result deserved re-examination. The first instance is discussed in his Chapter VI and refers to the case when alternatives are the possible distributions of goods in an economy, agents are individualistic (selfish) and it is socially desirable to rank states according to the Pareto principle. The second and third instances are addressed in Chapter VII, under the general heading of "similarity as the basis of social welfare judgments", but refer to different situations. One of them is the case where preferences over alternatives are single peaked, as discussed by Black a few years before Arrow's work. Here similarity refers to the fact that different agents use a common dimension to form their preferences, but these can vary across individuals. In the other case, that Arrow calls the idealist position, all agents share 
the same preferences.

In this Section we briefly discuss Arrow's and some other contributions to the discussion of these three sources of domain restrictions and their consequences. Two additional Sections 3 and 4, will be devoted to discuss in more detail some of the work on economic and political domains, and on single peakedness, before we turn to additional sources of questions and developments in Sections 5 and 6.

Before we elaborate on these three avenues opened by Arrow, we must briefly comment on an early and highly relevant paper by Julian Blau (1957). Blau discovered that Arrow's Condition 1, requiring the existence of one free triple was in fact insufficient to carry the conclusion of his theorem when more that three alternatives were at stake. This was an important finding, because it showed that Arrow's initial formalization of the idea that domains had to contain "a wide range of sets of individual orderings" needed adjustment, and so did the proof of his theorem. Blau provided these adjustment by requiring that all triples should be free, and referring to what we now know as the universal domain assumption as a particular instance where this assumption would hold. In his 1963 edition Arrow used the condition of universal domain, which has become standard, even if not necessary to precipitate the dictatorship conclusion, as we shall see. From here on, we use the universal domain as a reference point, and thus treat any departure away from as a domain restriction. But notice that the notion of a free triple has continued to prove important in different contexts, as it allows to express conditions on domains leading to both possibility and impossibility results. It will appear in several instances throughout our text. Very lucid accounts of its role in connection to the further study of social welfare functions are found in Maskin (1979) and Schmitz (1977).

Let us first comment on the consequences of specializing the analysis to focus on situations where alternatives represent possible states of an economy. Formally, a first step in that direction requires to identify alternatives as vectors of real numbers representing what each agent will obtain of each one of the goods in that economy. Arrow plunged directly into the case where goods are private, hence allocations for each agent may differ. That allowed him to discuss the individualistic assumption that agents are selfish. That case was particularly relevant because a main purpose of Social Choice and Individual Values was to provide new light in the complex disputes between economists of the time regarding welfare economics, its purposes and instruments. Chapters III and IV, which precede the statement and proof of his theorem, are indeed devoted to prepare the reader to enter that debate, where his decisive contribution was far from generating immediate consensus.

Indeed, the idea of a Bergson-Samuelson social welfare functions was strongly rooted in the welfare economics of the time (Bergson, 1938, Samuelson, 1947), and the question whether Arrow's result had any bearing on that concept was disputed by different economists, most notably by Samuelson (see Igersheim, 2017, for an account of the discussions that ensued).

Because of his special interest in the debate about the foundations of welfare economics, Arrow immediately considered the impact of his work on the analysis of economies with private goods and selfish consumers. The individualistic assumption is relevant in many economic situations. But there are other cases of interest in economics, involving the choice of levels for public goods, and in politics, where alternatives are the characteristics of candidates 
or platforms, that share an important common feature with private goods economies: all these social decisions can be modeled as choices of vectors of real numbers. In view of this fundamental analogy, we shall discuss all these models synthetically in the next section.

But let us go back to how Arrow defended of his impossibility in economic domains where agents are selfish and the Pareto principle must prevail, based on the following idea. He argued that any social welfare function satisfying these properties and the rest of his requirements in the impossibility theorem had to be the extension of a partial ordering which could be proven to precipitate dictatorship. Indeed, Blau's criticism to the original proof had to be taken into account to make this intuition work, and a great merit of Blau's paper is that he also provided an appropriate argument to that effect.

The second direction discussed by Arrow in the first part of his Chapter VII was that of escaping his impossibility result when Black's condition of single peakedness holds. Arrow stressed that this condition requires a similar assessment of the position of alternatives by different agents even if they finally do not agree on how to rank them. Under that condition, it is not only possible to obtain results regarding social welfare functions, but also extend them to the discussion of other types of social aggregators and to widely enlarge the range of questions in economic design admitting positive solutions. We shall devote Section 4 to explain how research has uncovered the many wonderful implications of single peakedness and related conditions like single crossing, top monotonicity, multidimensional or semi single peakedness, to answer in the positive not only Arrow's initial questions but also many others.

Yet a third direction is announced in the second part of his Chapter VII in Social Choice and Individual Values, when referring to domains of definition, and it comes with the discussion of the approach that Arrow calls the idealist position. He centers his attention on the views of different authors who assume, for one reason or another, that agents may be endowed with a common view regarding social alternatives. These include Rousseau, in reference to the notion of the general will, Kant on the role of the moral imperative, and later thinkers. Although he did not discuss it directly, the assumption that agents share the same view regarding what is correct, but differ in the information they have, is the starting point for the Condorcet jury theorem. In the historical remarks that Arrow added in his second edition he referred to the fact that Condorcet actually developed two different approaches. One led him to the discovery of cyclical patterns and the puzzles that Arrow himself was attacking in his work. He relates the other approach to the idealistic position, that he had associated in his first edition with Rousseau and Kant, this time establishing the connection between that line of thought with the theory of juries as developed by Condorcet, which he qualified as "a stochastic version of the idealistic position". We shall not pursue a discussion of this third topic in Arrow's list of subjects relating to domain restrictions, although we still think it would be of great interest to keep trying some sort of rejoinder between these two important lines of thought. Relevant questions regarding this framework, which appears to be orthogonal to the Arrowian one, have been the subject of much recent research. See, for example, the literature on communication in juries, as exemplified by the work of Austen-Smith and Banks (1996) and Austen-Smith and Feddersen (2005, 2006), or the writings on judgment aggregation, that are surveyed in List and Polak (2010). In spite of essential differences, the issue of domains is also important in these other cases. We insist on that point in our final remarks. 


\section{Economic and political domains}

In many occasions, it is natural to endow the set of alternatives with a specific structure, and then use the characteristics of the space of alternatives to generate domain restrictions whose interpretation arises naturally from the meaning attached to those alternatives in concrete applications.

Economic and political environments are clear examples where such possibilities arise. Vectors of real numbers stand for quantities of goods or candidates' characteristics, and this endows the space of alternatives with a lot of structure, allowing to introduce formal and economically meaningful restrictions on the sets of admissible preferences of agents (like selfishness or monotonicity), and on the functions used to aggregate them (like continuity).

The treatments of environments with private or public goods are different because some of the natural restrictions in one case and the other are not the same. But others are common, like those arising from the structure of alternatives as vectors of real numbers. We cannot be exhaustive, and will just try to give a taste of the questions raised and partially solved in these particular setups. Similar questions have been also discussed in other settings.

The early realization that impossibilities could persist even in the presence of domain restrictions led to the study of two complementary questions. One is to determine how much one can restrict the domains of definition of social welfare functions and still have the rest of Arrowian conditions to precipitate dictatorship. Such domains are referred as being Arrow inconsistent. The other is to identify domains that are as large as possible and still avoid the impossibility result. Ideally, both approaches would merge if one could fully characterize

the frontier between possibility and impossibility, but this is more a guide than a feasible objective.

These two quests generated a lot of research, but we shall only comment on a few papers and refer the interested reader to an extensive and masterful survey due to Le Breton and Weymark (2010), to Chapter 6 of Gaertner (2001) and Chapter 5 in McKelvey (1996).

The possibility of finding positive results for large domains, once properly restricted, was studied from different angles by Maskin (1979), Muller (1982) and Fishburn (1997, 2002), who also surveyed other works regarding the largest domains under which majority rule would not cycle.

On the other hand, negative results regarding the specific formulations of economic domains with private goods, were shown to persist, as in Border (1983). Kalai, Muller, and Sattertwaite (1979) concentrated in identifying sufficient conditions for a preference domain to be Arrow inconsistent. Their paper defined the notion of saturating preferences for economies with public goods, and was later extended to cover other cases.

Let us start by introducing some minimal formalism.

Let $N$ be a set of agents, $A$ be the set of alternatives, and let $\mathcal{B}$ denote the set of all reflexive and complete binary relations on $A$. Given $R \in \mathcal{B}$, we will denote by $P$ the induced strict relation such that $x P y$ if and only if $x R y$ and not $y R x$. A preference relation $R \in \mathcal{B}$ is transitive if for any triple $x, y, z \in A, x R y$ and $y R z$, implies $x R z$. It is a linear order if in addition to transitivity it is antisymmetric. $R$ is quasi transitive if its induced strict binary relation is transitive. Let $\mathcal{R} \varsubsetneqq \mathcal{B}$ be the set of all complete, reflexive, and transitive binary relations. 
The preferences of individuals over alternatives are represented by transitive, complete, and reflexive binary relations on $A$ : For each $i \in N$, let $\mathcal{R}_{i} \subseteq \mathcal{R}$ be the class of $i$ 's admissible preferences. We call $\mathcal{R}_{i}$ the domain of individual preferences and $\times_{i \in N} \mathcal{R}_{i}$ the domain of preferences. A preference aggregation rule on $\times_{i \in N} \mathcal{R}_{i}$ is a map $F: \times_{i \in N} \mathcal{R}_{i} \rightarrow \mathcal{B}$. A social welfare function on $\times_{i \in N} \mathcal{R}_{i}$ is a function $f: \times_{i \in N} \mathcal{R}_{i} \rightarrow \mathcal{R}$. Note that this is the notion we used without definition in the preceding section. A social choice function on $\times_{i \in N} \mathcal{R}_{i}$ is a function $f: \times_{i \in N} \mathcal{R}_{i} \rightarrow A{ }^{1}$

A free triple is a set of three alternatives such that everyone's preferences are unrestricted on it (that is, all possible orderings over the three alternatives are admissible in each agent $i$ 's set of preferences). A preference domain $\times_{i \in N} \mathcal{R}_{i}$ is saturating if any two pairs of alternatives are connected through a sequence of free triples. ${ }^{2}$

The idea of connecting different pairs of alternatives relative to a domain through a systematic procedure will be highlighted in Section 5 as a productive source of results, both positive and negative. We postpone discussion of the general idea, but wanted to mention here that, again, Arrow's free triple condition is at the root of this form of thinking.

Kalai, Muller, and Satterthwaite (1979) prove that any social welfare function with a common saturating preference domain satisfying Independence of Irrelevant Alternatives (IIA) and Weak Pareto (WP) must be dictatorial.

Further work by Bordes and Le Breton (1989) proved an analogous result through the use of a new concept of hypersaturating preferences, which applies in particular to the $n k$ dimensional space of standard preference profiles with private goods, among other domains.

Le Breton and Weymark (2010) show that for a Cartesian set of alternatives $A$, if a social welfare function on a preference domain that is both selfish and hypersaturating satisfies IIA and WP, then it is dictatorial.

The research on economic domains by these authors, and also by Maskin (1976) and Border (1983), exhibited a wide array of cases where Arrow's dictatorship conclusion holds. The analysis can be further extended to cases where feasibility constraints rule out the frequent assumption that domains are Cartesian products, like, for example, when alternatives are allocations in an Edgeworth box (Bordes and Le Breton, 1990; Bordes, Campbell and Le Breton, 1995).

The research on Arrow inconsistent environments, did also provide examples in the opposite direction, by identifying cases where even slight variations in the definition of such environments allow to escape the dictatorship conclusion. However, the functions that avoid full dictatorship are in general quite unattractive, as they retain partial dictators.

Another consequence of modelling alternatives as vectors of real numbers is that the relevant spaces on which preferences and social welfare functions are defined become topological, whose characteristics can then be used to raise and solve additional questions. Several authors have exploited the richness of the set of preferences to show how impossibilities evolve

\footnotetext{
${ }^{1}$ Notice that many other variants of collective rules could be adopted when defining how individual preferences are combined. Domains could be enlarged by not requiring individual preferences to be transitive, and codomains could allow for different expressions of aggregation, including sets of alternatives, choice functions, lotteries, or different sorts of binary relations. We restrict attention to the three basic forms defined above, which are enough to make our points.

${ }^{2}$ We refer the reader to Le Breton and Weymark (2010) for definitions and results related to this section.
} 
when enlarging the set of alternatives to be a continuum, rather than discrete. Kramer (1973) pioneered in that direction, by showing how easily new cyclical patters can emerge in that case. In a line of work that exploits the topological structure of the set of alternatives more deeply, Redekop (1991) proved that Arrow inconsistency is retained under domain restrictions that preserve a large enough degree of preference diversity, in a precise sense.

Specifically, let $X=\mathbb{R}_{+}^{m}$ and $\mathcal{C}_{c m}$ be the set of all preference orderings on $X$ that are continuous and monotonic. Le Breton and Weymark (2010) show that if $X=\mathbb{R}_{+}^{m}$ with $m \geq 2$ and $\mathcal{D}_{*} \subseteq \mathcal{C}_{c m}$ is somewhere dense, then there is no social welfare function $F: \mathcal{D}_{*}^{n} \rightarrow \mathcal{R}$ that satisfies IIA and WP except for dictatorial rules.

Another set of important contributions that exploits the topological structure of the social choice problem was sparkled by the work of Chichilnisky (1980, 1982). Her ambitious plan was to reformulate the issues considered by social choice theory in a form that would allow comparisons and unification with market models. The approach proposes the abandonment of the Arrowian condition of independence of irrelevant alternatives, and stresses the decisive impact of continuity and contractibility in drawing the line between unified models that admit possibility results and others where impossibilities prevail.

We wanted to call the attention of the reader to this line of research but will not follow it up further, as it escapes a bit the standard formulation of social choice. The interested reader is referred to the survey by Baigent (2010), the outline in Gaertner (2001) and Chichilnisky and Heal (1983).

Last, but not least, let us mention that what we understand here the public goods model is in fact the setting for much of the voting theory developed by political scientists, under the assumption that alternatives are real valued vectors indicating the degree of compliance with different characteristics, voters have a single preferred alternative or bliss point and preferences decline as the alternatives are "further away" from that ideal.

Again, there is an extensive literature on the subject, whose essence consists in showing that the possibility of cyclical patters is pervasive, not only under majority voting but also when other rules based on binary comparisons are considered. Chaos theorems have been refined to prove the extent to which cycles, who are at the root of Arrow's impossibility result, are to be expected to arise in this class of models, unless very restrictive assumptions are made (see Plott, 1967, Davis, De Groot, and Hinich, 1972, for early contributions, and McKelvey, 1996 or Schofield, 1978 for accounts by fundamental contributors to this line of research).

In this section we have concentrated mostly on papers devoted to the analysis of Arrowian social welfare functions. But the literature has evolved in different directions, and a large part of papers in social choice theory formalize the outcome of social decision processes not as an aggregate preference through which to select alternatives, but directly as choices of one or several alternatives. Attention has somewhat shifted from social welfare functions to social choice functions or correspondences, especially since the analysis of the latter was proven, through the work of Gibbard and Satterthwaite (see Gibbard, 1973 and Satterthwaite, 1975), to be the most appropriate formalism to discuss strategic issues.

In the next section we mix the discussion of social welfare functions with that of social choice functions, which take the central position in Section 5. 


\section{The wonders of single peaked domains}

We already mentioned that the consequences of restricting attention to single peaked preferences were extensively discussed by Arrow in connection to his impossibility result. In fact, considering this domain condition has proven to be, since then, an extremely fruitful idea, which can be factorized into different properties, extended in several ways, and related to other interesting and natural domain restrictions. We shall discuss several lines of research, reaching to the present, which prove its importance in different applications: location of public services, choice of political candidates, allocation of funds, election of members to a club, etc.

In this section we restrict our attention to the case where both the set of alternatives and agents is finite, and preferences are linear orders. Elements in $\mathcal{R}^{n}$ are called preference profiles denoted by $R_{N}=\left(R_{1}, \ldots, R_{n}\right)$. For any $i \in N$, let $\tau\left(R_{i}\right)$ denote the best alternative of $R_{i}$ on $A$, also called its peak.

A preference profile $R_{N}$ is single peaked if there exists a linear order $\prec$ on $A$ such that for each agent $i \in N$ and any two alternatives $x, y \in A, \tau\left(R_{i}\right) \prec x \prec y$ or $y \prec x \prec \tau\left(R_{i}\right)$, then $x P_{i} y$.

\section{Characterizing single peakedness}

In many applications, the order relative to which single peakedness is predicated arises naturally from the interpretation of the situation that is modeled. This is the case, for example, when alternatives are political candidates, whose position on a left-right spectrum is agreed upon by all voters, or locations of some public facility on a linear space. In other cases, determining the existence of an order that turns the profile into a single peaked one is itself a question that needs analysis. Hence, one may ask whether there are properties that characterize single peakedness without need to explicitly refer to the underlying order. Indeed there are two, that were discovered with a considerable time gap between them.

One condition was identified in seminal papers by Sen (1966) and Sen and Pattanaik (1969) and it involves the ranking of triples of alternatives by triples of agents.

A preference profile $R_{N}$ satisfies Condition 1 if for any three agents and each triple of alternatives, there exists one alternative that no agent ever ranks as being worse than the other two.

Condition 1 is necessary for a profile to be candidate to satisfy single peakedness, but not sufficient. Actually, it is one of three conditions that Sen and Pattanaik collected under the common name of value restriction.

Much more recently, Ballester and Haeringer (2011) identified a second necessary condition, this time involving four alternatives, but only two agents at a time.

A preference profile $R_{N}$ satisfies Condition $\mathbf{2}$ if for any two agents $i$ and $j$, and every four alternatives $x, y, z, w$ such that $x P_{i} y P_{i} z$ and $z P_{j} y P_{j} x$, it cannot be that $w P_{i} y$ and $w P_{j} y$.

These two authors prove that Conditions 1 and 2, together, characterize single peaked preference profiles. This result nicely closes a gap in our understanding of single peakedness.

\section{Aggregation and decision under single peakedness}


Consider the following frameworks, that will allow us to recall the best known consequences entailed by single peakedness, and to raise additional questions.

An immediate consequence of assuming that all preference profiles in the domain of an aggregation rule are single peaked is that the social preference obtained by simple majority voting is transitive, when the number of agents is odd, and quasi transitive in all cases. Since majority voting satisfies all other conditions demanded by Arrow's impossibility theorem, restricting preferences to be single peaked allows us to escape from the impossibility. In fact, many other nice aggregation rules can also be defined in single peaked domains. See AustenSmith and Banks (1996), for example. Regarding the construction of social welfare functions, a remarkable characterization under a weak version of single peakedness is provided in Ehlers and Storcken (2008).

A very classical observation regarding majority voting as a social choice function is that for all single peaked preference profiles, it selects the median of the peaks' distribution whenever this is unique. This median voter result is extremely useful to analyze political and location problems, among other applications.

Since single peakedness is such a fruitful condition on domains and leads to nice positive results, it is natural to ask whether some alternative condition on preference profiles may lead to similar conclusions.

Recall that Arrow stressed that single peakedness relates the agents' preferences to an underlying onedimensional ranking, that expresses a similarity of their views regarding alternatives, even if they value them differently. That led him to conjecture that similarity among agents is at the root of the solution to the aggregation problem.

The attractiveness of single peakedness does not only come from the technical facts regarding the possibility of designing mechanisms with good properties when preferences satisfy it. It also derives from the fact that one can present that condition as rather possible in a variety of cases and under different interpretations. Single peaked preferences on an interval can be the result of agents' tendency to prefer public services as near as possible to their own location, or to prefer candidates whose one dimensional views are closer to their own, or as a reduced form of the trade-off between the values of two competing uses of resources when actual preferences on pairs are convex. Another domain restriction that entails a natural justification is single dipped preferences: there, agents prefer locations (typically of public bads) to be as far as possible from their own, or have a preference for extremist candidates. Other types of restrictions, as we shall see, are harder to justify, though in each case it is possible, and relevant to look for reasons to use them, other than the fact that "they work". A different form of similarity among preferences underlies the definition of another important domain restriction:

A preference profile $R_{N}$ satisfies single crossing if there exist a linear order $\succ$ on the set of alternatives and a linear order $\succ^{\prime}$ on the set of agents such that for all $i, j \in N$ such that $j \succ^{\prime} i$, and for all $x, y \in A$ such that $y \succ x$, if $y P_{i} x$ then $y P_{j} x$.

The implications of single crossing on the design of aggregation and decision rules is quite parallel to those of single peakedness. For an odd set of agents, the preference of the median voter, according to the reference ranking of agents, actually coincides with the majoritarian social preference and is thus transitive. And the top alternative for this median agent is the 
majority winner. Again, slight qualifications must be added when the number of voters is even. Thus, single crossing performs very well. ${ }^{3}$

Given the similarities of results, one could ask whether single peakedness and single crossing are somewhat part of the same family of domain conditions. Indeed, they are.

In Barberà and Moreno (2011) it is proven that, in fact, there is a common root: a weaker condition, called top monotonicity, can be imposed on domains, is implied by both single peakedness and single crossing, and still retains the common property that a median voter is well defined and would choose the majoritarian outcome.

A preference profile $R_{N}$ is top monotonic if there exists a linear order $\succ$ over $A$, such that for each agent $i \in N$, there exists $\tau\left(R_{i}\right) \in A$, and for all $i, j \in N$, any $z \in A$ such that $\tau\left(R_{i}\right) \succ \tau\left(R_{j}\right) \succ z$ or $z \succ \tau\left(R_{j}\right) \succ \tau\left(R_{i}\right)$ then $\tau\left(R_{j}\right) P_{i} z$.

We can now state the following result which is a corollary of Theorem 1 in Barberà and Moreno (2011) for strict preferences and when the aggregation rule is majority voting: if the profile of preferences $R_{N}$ satisfies top monotonicity, then the median(s) of the distribution of agents' peaks coincides with the majoritarian outcome(s).

Until here we have spoken of single peakedness as a property of single profiles, but it may be worth defining what we mean by a single peaked domain. And here we have two choices, both of them interesting. One of them is to simply define a domain to be single peaked if each one of the profiles it contains is single peaked relative to some order of the alternatives, not necessarily the same for all profiles. We can label such domains as extensively single peaked. A more restricted possibility is to consider that all the profiles in a single peaked domain must satisfy this condition with respect to the same order of alternatives. The results we have presented till now apply to the extensive definition, but from now on we will concentrate on the narrower one, which requires us to assume a common order.

\section{Single peakedness and strategy proofness}

The interesting facts about single peakedness do not stop at considering classical Arrowian aggregation issues. One important aspect that Arrow mentioned insightfully but did not develop in his book was the question of strategic voting.

A social choice function $f$ on $\times_{i \in N} \mathcal{R}_{i}$ is manipulable at $R_{N} \in \times_{i \in N} \mathcal{R}_{i}$ by coalition $C \subseteq N$ if there exists $R_{C}^{\prime} \in \times_{i \in C} \mathcal{R}_{i}\left(R_{i}^{\prime} \neq R_{i}\right.$ for all $\left.i \in C\right)$ such that $f\left(R_{C}^{\prime}, R_{N \backslash C}\right) P_{i} f\left(R_{N}\right)$ for all $i \in C$. A social choice function is group strategy proof if it is not manipulable at any $R_{N}$ by any coalition $C \subseteq N$, and it is (individually) strategy proof if it is not manipulable by any singleton agent.

Following Gibbard and Satterthwaite's initial negative result on the possibility of designing nontrivial strategy proof rules, several fruitful lines of research on the design of strategy proof rules did concentrate on relaxing the universal domain assumption, which clearly played a crucial role in precipitating their conclusion. And single peaked domains also turned out to be crucial for making it possible to define social choice functions with

\footnotetext{
${ }^{3}$ Single crossing is equivalent to order restriction, a condition also used in different contexts. See Gans and Smart (1996).
} 
good incentive properties. All the comments that ensue refer to domains of single peaked preferences that share the same underlying order of alternatives.

Let us start by an important remark. Under single peaked preferences the social choice function generated by majority voting ${ }^{4}$ satisfies not only the property of strategy proofness but also that of group strategy proofness. And again, this is only one of the many interesting functions that can be defined having these properties on the domain of single peaked preferences.

A fundamental paper by Moulin (1980) characterized all the rules in this domain that are strategy proof and unanimous (i.e., whenever all agents agree on what alternative is their best then this is the social choice). Without loss of generality, we can identify the finite set of ordered alternatives with integer numbers. Then, the class of rules satisfying those properties coincide with that of minmax rules, defined as follows.

A social choice function $f$ is a minmax rule associated with a set of integers $\left(a_{S}\right)_{S \subseteq N}$ if for each preference profile $R_{N}, f\left(R_{N}\right)=\min _{S \subseteq N}\left\{\max _{i \in S}\left\{a_{S}, \tau\left(R_{i}\right)\right\}\right\}$.

Massó and Moreno de Barreda (2011) showed that additional rules can satisfy the same properties if we further restrict the class of single peaked preferences by using symmetry conditions that are natural in many instances. Notice that here we refer to results which apply to single peaked domains in the version whereby all profiles in the domain must satisfy the condition for the same underlying order of alternatives. In an interesting paper, Penn, Patty, and Gailmard (2011) have remarked that a notion of group strategy proofness could not be attained if we considered the extensive definition of a single peaked domain. These authors make the point that certain domains that avoid aggregation issues may not solve incentive problems. Notice that under the extensive definition of single peaked domains, Arrow's idea of similarity among agents is weakened, while assuming that all profiles in the domain share the same underlying order of alternatives expresses a much stronger notion of similarity. In fact, when all profiles in a single peaked domain arise from the same linear order of alternatives, it makes sense to think of single peakedness as a property of individual preferences (relative to that common order). This allows us to define the domains of single peaked profiles as a Cartesian product, and to avoid any difficulties in interpreting the meaning of strategy proofness.

\footnotetext{
${ }^{4}$ Here again this loose statement should be qualified depending on whether the number of alternatives is odd or even. The precise statement of Moulin (1980) will clarify any possible ambiguity. Similar remarks regarding strategy proofness of the median rule under single crossing preferences is contained in Saporiti and Tohmé (2006).
} 


\section{Extensions of single peakedness}

In view of the fruitful implications of single peakedness there have been many efforts to adapt this idea to different contexts in order to address a variety of questions.

Demange (1982) introduced a natural extension of the unidimensional property of single peakedness to preference profiles that satisfy a similar condition relative to a tree, and showed that aggregation difficulties could still be circumvented under this less demanding requirement.

Chatterji, Sanver, and Sen (2013) adapt her notion of single peakedness and introduce the concept of semi single peakedness assuming that the set of individual preferences is the same for all agents and it is a subset of linear orders.

Let $G$ be a tree, that is a graph where there is a unique path linking every pair $a_{j}, a_{k} \in A$. A path is maximal if it cannot be "extended" by adding more edges at the ends. Denote $P(G)$ as the set of maximal paths in $G$. For $p_{t} \in P(G)$ and $a_{k} \in A, a_{k} \notin p_{t}$, let $\gamma\left(p_{t}, a_{k}\right)$ be the unique alternative, say $a_{r}$, in $p_{t}$ with the property that every path from an alternative in $p_{t}$ to $a_{k}$ contains $a_{r}$. The map $\lambda: P(G) \rightarrow A$ is a threshold assignment map if there exists $a_{k} \in A$ such that: (i) for all $p_{t} \in P(G),\left[a_{k} \in p_{t}\right] \Longrightarrow\left[\lambda\left(p_{t}\right)=a_{k}\right]$, and (ii) for all $p_{t} \in P(G)$, $\left[a_{k} \notin p_{t}\right] \Longrightarrow\left[\lambda\left(p_{t}\right)=\gamma\left(p_{t}, a_{k}\right)\right]$.

For any pair of alternatives $a_{j}, a_{k} \in A$, let $\left\langle a_{j}, a_{k}\right\rangle$ denote the unique path connecting $a_{j}$ and $a_{k}$.

The domain of individual preferences $\mathcal{R}_{i}$ is semi single peaked with respect to the admissible pair $(G, \lambda)$ if for all $R_{i} \in \mathcal{R}_{i}$ and all $p_{t} \in P(G)$ such that $\tau\left(R_{i}\right) \in p_{t}$, we have: (i) $\left[a_{r} \in p_{t}\right.$ such that $\left.\lambda\left(p_{t}\right) \in\left\langle\tau\left(R_{i}\right), a_{r}\right\rangle\right] \Longrightarrow\left[\lambda\left(p_{t}\right) P_{i} a_{r}\right]$, and (ii) $\left[a_{r}, a_{s} \in p_{t}\right.$ such that $a_{r}, a_{s} \in\left\langle\tau\left(R_{i}\right), \lambda\left(p_{t}\right)\right\rangle$ and $\left.a_{r} \in\left\langle\tau\left(R_{i}\right), a_{s}\right\rangle\right] \Longrightarrow\left[a_{r} P_{i} a_{s}\right]$.

Chatterji, Sanver, and Sen (2013) have proven that if a domain (of individual preferences) is semi single peaked, then there exists an anonymous, strategy proof, unanimous, and tops only (that is, social outcomes depend only on agents' best alternative) social choice function on that domain.

Introducing indifferences in the definition of single peakedness is a delicate matter. Allowing for several alternatives to be tied in the top (see Fishburn, 1973, Moulin, 1984, and Berga, 1998) or having at most two indifferent alternatives, one on each side of the peak, is not a problem. Yet, other types of indifferences can cause the breakdown of all the nice results that are guaranteed by single peakedness (see Barberà, 2007). In the case of private goods and selfish preferences, indifferences between alternatives where one agent receives the same are unavoidable, but then it is possible to still require that their preferences on private consequences be single peaked, and to obtain nice results regarding the existence of strategy proof mechanism (Sprumont, 1991 and Barberà, Jackson, and Neme, 1997).

In many applications, alternatives can be expressed as vectors of characteristics. A natural direction to extend the notion of single peakedness is the one taken in political science, when assuming that alternatives are some subset of $\mathbb{R}^{n}$, and agents' preferences have a maximal alternative and are concave. In that case, important results, exemplified by McKelvey's (1976) and Schofield's (1978) chaos theorems have proven that such an extension 
does not avoid cyclical patterns, for majority or for other rules, and that Arrow's impossibility still prevails.

By contrast, an alternative extension of the notion of single peakedness still provides good results regarding individual incentive properties. Let us be more specific.

Consider alternatives to be elements of the Cartesian product of $K$ integer intervals $A=\prod_{k=1}^{K} B_{k}$, where $B_{k}=\left\{a_{k}, \ldots, b_{k}\right\}$, for any $k=1, \ldots, K$ and endow this set with the $L 1$ norm where $\|x\|=\sum_{k=1}^{K}\left|x_{k}\right|$. Given $\alpha, \beta \in A$, the minimal box containing $\alpha$ and $\beta$ is defined by

$$
M B(\alpha, \beta)=\{\gamma \in A:\|\alpha-\beta\|=\|\alpha-\gamma\|+\|\gamma-\beta\|\}
$$

A preference $R_{i} \in \mathcal{R}$ is (multidimensional) single peaked if it has a unique maximal element $\tau\left(R_{i}\right) \in A$, and for any $\gamma, \beta \in A$, if $\beta \in M B\left(\gamma, \tau\left(R_{i}\right)\right)$ then $\beta R_{i} \gamma$.

Notice that the distance between any two alternatives $\alpha$ and $\beta$ is the length of any shortest path between $\alpha$ and $\beta$ and the extension of single peakedness is based on the idea that one alternative is better than another if it is "closer" to the best.

In multidimensional single peaked domains, there exist many nontrivial strategy proof rules. For example, one can ask agents to reveal their best alternative, decompose this information by projecting each component of this bliss point on each of the integer intervals, choose the median of these projected best components in each interval, and finally propose the vector of these onedimensional medians as the social outcome. ${ }^{5}$

Yet, there are losses in that generalization. A major difference between the onedimensional and the multidimensional world is that, in the former, strategy proofness implies group strategy proofness as well, and thus efficiency. This implication fails in the multidimensional case.

Notice that the positive results one obtains under the notion of multidimensional single peakedness do not hold if we extend single peakedness by using the Euclidean metric, as political science's canonical model does. Also remark that, even under multidimensional single peakedness, which allows for positive results regarding strategy proofness, one can get no parallel when dealing with the aggregation problem: McKelvey and Schofield's chaos theorems, whereby cycles persist in these worlds, are not overruled by altering the metric.

\section{What domains admit strategy proof rules?}

Another intriguing question remains, once having proved that multidimensional single peaked domains admit many strategy proof mechanisms to be defined on them. Is it possible,

\footnotetext{
${ }^{5}$ For precise characterizations and properties of these rules in Cartesian domains, see Barberà, Gul, and Stachetti (1993), Le Breton and Sen (1995 and 1999). The positive results in these papers must be qualified when some potential alternatives cannot be chosen and the range of the function is not a Cartesian product. See Barberà, Massó, and Serizawa (1998), Barberà, Massó, and Neme (1997), and Barberà, Massó, and Neme (2005).
} 
in some sense, that this condition on domains is not only sufficient but also necessary to achieve strategy proofness? Several authors have tried to substantiate the hunch that some form of single peakedness lures behind the possibility to design satisfactory strategy proof rules, and have gotten quite close to it. In particular, Chatterji, Sanver, and Sen (2013) show that for a wide class of domains that they call strongly path connected it is only possible to define anonymous, strategy proof, and unanimous social choice functions that depend only on the best alternative of agents if the domain of definition is semi single peaked on a tree. The result can be interpreted in spirit as partial proof that domains admitting "well behaved" social choice functions must be "close" to single peaked.

In a similar spirit Nehring and Puppe (2007) investigated the structure of strategy proof social choice functions in an abstract algebraic setting, and again showed the prominent role of median spaces, that can be viewed as an extension of single peakedness, as domains allowing for strategy proof rules.

In this section we have tried to emphasize how a condition that was recognized to be interesting long ago, and that is heavily used in different contexts of application to politics and public economics, has kept the interest of researchers alive over the years, and continues to prove essential in many contexts, and worth understanding in depth, like mechanism design (Roth, 2008), judgement aggregation (List, 2012), and even new applications (Gaertner, 2019).

\section{Connected paths as a basis for domain conditions}

It is not easy to classify domain conditions in a consistent and exhaustive way. Gaertner (2001) does not propose a systematic classification but distinguishes between different types of conditions for expository purposes. He studies those that apply to continuous spaces, those that are based on the quantitative distribution of preferences, and those that he calls exclusion conditions. Single peakedness belongs to this latter category. If it is defined relative to a fixed order then some preferences are excluded. If we do not fix the order a priori then any preference can be attributed to agents in a single peaked profile, but not all combinations of preferences are admissible: once some agents hold a preference, those of others may be restricted.

We want to present now a set of conditions on domains that adopt a different form than the ones we just mentioned. This will add variety to the already long list of conditions that have attracted the interest of analysts. We have found some of them to be useful in our own work, and we have also identified other pieces of research that use similar ideas. The common characteristic of the class of conditions we want to present is that they require domains to contain certain sequences of preference profiles, obtained by successive changes in preferences by a single agent at a time. The choice of those preferences that an agent can introduce at each step when she changes preferences depends on each particular condition, but can in all cases be indexed by an alternative, used as a reference point, that determine what preference changes are admissible. Hence, it is more precise to say that the conditions

are imposed on sequences of pairs, each one formed by one alternative and an admissible 
preference profile. ${ }^{6}$

Before presenting any specific condition in this class, let us refer to a fundamental technique that was developed by Alan Gibbard in his seminal paper on strategy proofness, and has since been at the basis of many proofs and lines of argument. Given two preference profiles, $R_{N}$ and $R_{N}^{\prime}$, Gibbard considered the sequence of profiles that are obtained by changing one by one the preferences of agents, starting at $R_{i}$ and leading to $R_{i}^{\prime}$.

This simple construction of a sequence connecting $R_{N}$ to $R_{N}^{\prime}$ was used by Gibbard (1973), and is the starting point for many arguments and proofs in the study of incentive properties. What matters to us here is that it is the seed for other similar constructions. Notice that under the universal domain assumption, all profiles in the sequence connecting any $R_{N}$ to any $R_{N}^{\prime}$ are also part of the domain. But this needs not be the case if some combinations of preferences are excluded from the domain. Then, determining whether certain connections can be established without leaving a given set of profiles, to be used as a domain, is essential to establish how far one can go in proving possibility or impossibility results. As an example, consider one of the uses that has this technique of travelling from one profile to another through a sequence that derives from changing one preference at a time.

Given a social welfare function $F$, two alternatives $x$ and $y$, and two preference profiles $R_{N}$ and $\widetilde{R}_{N}$ such that $F\left(R_{N}\right)$ ranks $x$ over $y$ and $F\left(\widetilde{R}_{N}\right)$ ranks $y$ over $x$, there must be some profile in the sequence we described above where the image of $F$ jumps and changes the ordering of these two alternatives. Similarly, if $f$ is a social choice function and $f\left(R_{N}\right)=x$, while $f\left(\widetilde{R}_{N}\right)=y$, there must be some profile $R_{N}^{\prime}$ in the path from $R_{N}$ to $\widetilde{R}_{N}$ where $f\left(R_{N}^{\prime}\right)$ differs from $x$ for the first time. This technique of gradual substitutions can be used to locate agents that are pivotal under a given rule, and are at the basis of direct proofs of both Arrow's and Gibbard-Satterthwaite's theorems that were first proposed in Barberà (1980, 1983).

The conditions on domains that we shall present are used in a similar manner, and require the possibility to study how social choice functions change values at critical points along a sequence. We present them one by one, in connection to different normative issues that the literature has considered and found solutions for by appropriately defining the relevant domains. All of them share similar characteristics, but are not identical, since they have been developed in connection to a variety of different questions.

\section{The frontiers between dictatorial and non dictatorial domains}

Here is a first set of questions that give rise to domain restrictions based on connections among different pairs formed by a feasible alternative and a preference profile in the domain.

As we have already remarked, one may want to relax domain conditions that are unnecessarily strong and play a role in impossibility results, in order to explore the frontier

\footnotetext{
${ }^{6}$ In his classical book, Fishburn (1973, page 178) proposed a classification of different conditions that one may impose on social choice functions, and distinguished, among others, between intraprofile and interprofile conditions, depending on whether the requirements on the outcomes of a function could be expressed in reference to one profile at a time, or needed to identify several ones that were somewhat connected. The conditions we present are on domains of definition, rather than on a function's outcome, and the classification does not directly apply, but there is a parallel. Single peakedness can be checked profile by profile, while the conditions we are about to present refer to combinations of profiles.
} 
between possibility and impossibility. Aswal, Chatterjii, and Sen (2003) have investigated what characterizes those domains that, even allowing for restrictions, still cannot avoid the conclusion that only dictatorial social choice functions can satisfy strategy proofness. Their result is based on the following definition of linked domains where they consider a common preference domain and linear orders.

For any $i \in N$, let $\tau_{s}\left(R_{i}\right)$ denote the $s$-th ranked alternative in $R_{i}$ on $A$ where $\tau_{1}\left(R_{i}\right)=$ $\tau\left(R_{i}\right)$.

A pair of alternatives $a_{j}, a_{k}$ are connected, denoted $a_{j} \sim a_{k}$ if there exist $R_{i}, \widetilde{R}_{i} \in \mathcal{R}$ such that $\tau\left(R_{i}\right)=a_{j}, \tau_{2}\left(R_{i}\right)=a_{k}, \tau\left(\widetilde{R}_{i}\right)=a_{k}$, and $\tau_{2}\left(\widetilde{R}_{i}\right)=a_{j}$. Also, an alternative $a_{j}$ is linked to $B \subset A \backslash\left\{a_{j}\right\}$ if there exist $a_{k}, a_{r} \in A$ such that $a_{j} \sim a_{k}$ and $a_{j} \sim a_{r}$. The domain of individual preferences $\mathcal{R}_{i}$ is linked if there exist a one to one function $\sigma: A \rightarrow A$ such that (i) $a_{\sigma(1)} \sim a_{\sigma(2)}$, and (ii) $a_{\sigma(j)}$ is linked to $\left\{a_{\sigma(1)}, a_{\sigma(2)}, \ldots, a_{\sigma(j-1)}\right\}, j=3, \ldots, m$.

Based on this condition, which substantially weakens the assumption of universal domain used in the Gibbard-Sattherthwaite theorem, they can prove that, still, any nonmanipulable social choice function defined on a linked domain must be dictatorial.

On the positive side, as we have already mentioned in the preceding section, Chatterji, Sanver, and Sen (2013) produced a theorem proving that it is not only possible to define satisfactory strategy proof rules under appropriate domains, but that a form of single peakedness, semi single peakedness, is essential to attain this objective. We already stated this result in the preceding section but here we want to point out that the additional condition of strong path connectedness they impose on domains to obtain the result fits into our category of connectedness requirements.

A pair of alternatives $a_{j}, a_{k}$ are strongly connected, denoted $a_{j} \approx a_{k}$ if there exist $R_{i}, \widetilde{R}_{i} \in \mathcal{R}$ such that $\tau\left(R_{i}\right)=a_{j}, \tau_{2}\left(R_{i}\right)=a_{k}, \tau\left(\widetilde{R}_{i}\right)=a_{k}, \tau_{2}\left(\widetilde{R}_{i}\right)=a_{j}$ and $\tau_{s}\left(R_{i}\right)=\tau_{s}\left(\widetilde{R}_{i}\right)$ for all $s=3, . ., m$. The domain of individual preferences $\mathcal{R}_{i}$ is strongly path connected if for all $a_{j}, a_{k} \in A$, there exists a sequence of alternatives $a_{s(r)} \in A, r=0, \ldots, T$, such that (i) $a_{s(0)}=a_{j}$, (ii) $a_{s(T)}=a_{k}$, and (iii) $a_{s(r)} \approx a_{s(r+1)}, r=0, \ldots, T-1$.

This line of work is still very active, as witnessed, among others, by the recent work of Chatterji and Massó (2018). It is clear that most of the proposed domain restrictions we have discussed have a technical flavor, while a few, notably single peakedness are easier to motivate through real life examples. But let us elaborate a bit on the meaning and the motivation of the connectedness restrictions. Essentially, they require that the different profiles are linked through changes that lead from one admissible profile to another which is also permissible. This allows us to argue that the impact of domain restrictions is not so much quantitative as it is structural. It is not the number of preference relations that counts to determine the frontier between possibility and impossibility results, but rather the structure of the connections between different admissible environments.

We can go even further and propose an interpretation that is still tentative but hopefully suggestive. We refer to the notions of knit and partially knit environments (Barberà, Berga, and Moreno, 2018). Knit domains lead to strong impossibilities, partially knit ones admit nice rules, and since the restrictions satisfied by these two types of environments do not differ 
much, one could say that they both lie close to the frontier between the two worlds that the literature about domain restrictions tries to explore. Remember that Arrow interpreted single peakedness as a form of agreement among individuals: agents may differ in preferences, but they agree on a common underlying order of alternatives. By analogy, a main difference between environments that are knit and others that are not is related to the following fact: it is necessary for an environment to be knit that the changes in the information of an agent that carry an improvement of some alternative, imply changes in the preferences of some other for that alternative in the opposite direction. Hence, knitness introduces the possibility of strong disagreement among individuals when evaluating situations, while environments that are not knit imply that some form of basic collective agreement.

At any rate, these last remarks can only reinforce our message that there is still much to do in the trail the Arrow opened for us.

\section{Some equivalence results}

We shall now describe several questions regarding the connections between different normative requirements of interest, and how these depend on the domain of definition of the rules on which these requirements are predicated.

Let us begin by one question, having to do with the possibility that strategy proofness might be characterized by some elementary requirements. ${ }^{7}$

The following two very simple conditions are necessarily satisfied by strategy proof social choice functions. One is a very sharp version of monotonicity: if $f\left(R_{N}\right)=x$, and $R_{N}^{\prime}$ is a profile where, "ceteris paribus", $x$ is now better ranked by some agents than at $R_{N}$, then $f\left(R_{N}^{\prime}\right)=x$ as well. The other condition requires that, if $f\left(R_{N}\right)=x$, and the upper and lower contour sets of $x$ in $R_{N}^{\prime}$ are the same as the ones in $R_{N}$, again $f\left(R_{N}^{\prime}\right)$ must equal $x$. These two necessary conditions are, however, not always sufficient to precipitate strategy proofness, depending on the domain of definition of the social choice function where they apply. In Barberà, Berga, and Moreno (2012) we show that they do if the domain is intertwined, a condition that we now describe for strict preferences over consequences.

For any $i \in N$, let $B_{i}$ be a non-empty set of possible consequences for $i, A \subseteq \times_{i \in N} B_{i}$ be the set of alternatives, and, abusing notation, let $\mathcal{R}_{i}$ be the set of agent $i$ 's preferences on $B_{i}$. We assume that preferences are selfish, that is, for all $z, x \in A, z R_{i} x$ if and only if $z_{i} R_{i} x_{i}$.

Let $R_{i}, \bar{R}_{i} \in \mathcal{R}_{i}, y \in A$. We say that $\bar{R}_{i}$ is a $y$-direct transform of $R_{i}$ if either (i) for any $v \in A,\left[y P_{i} v \Leftrightarrow y \bar{P}_{i} v\right]$, or else (ii) for any $v \in A,\left[y P_{i} v \Rightarrow y \bar{P}_{i} v\right]$, and for any $w, t \in A \backslash\{y\}$, $\left[w P_{i} t \Leftrightarrow w \bar{P}_{i} t\right]$.

Let $R_{i}, \bar{R}_{i} \in \mathcal{R}_{i}, y \in A$. We say that $\bar{R}_{i}$ is a $y$-transform of $R_{i}$ if there exists a finite chain of preferences, say $\left\{R_{t}\right\}, t=1, \ldots, k$, such that $R_{1}=R_{i}, R_{k}=\bar{R}_{i}$ and for any $t>1, R_{t}$ is a $y$-direct transform of $R_{t-1}$.

Let $R_{i}, R_{i}^{\prime} \in \mathcal{R}_{i}, z, x \in A$ where $z P_{i} x$. We say that $R_{i}$ is $(z, x)$-intertwined with $R_{i}^{\prime}$ if there exists $\bar{R}_{i} \in \mathcal{R}_{i}$ such that $\bar{R}_{i}$ is both an $x$-transform of $R_{i}$ and a $z$-transform of $R_{i}^{\prime}$.

\footnotetext{
${ }^{7}$ A presentation of some of the properties that follow, directed to a computer science audience, is contained in Barbera, Berga, and Moreno (2013).
} 
A set of individual preferences $\mathcal{R}_{i}$ is intertwined if for any $R_{i} \in \mathcal{R}_{i}$, for any $z, x \in A$ such that $z P_{i} x$, and any $R_{i}^{\prime} \in \mathcal{R}_{i}, R_{i}$ is $(z, x)$-intertwined with $R_{i}^{\prime}$. A domain $\times_{i \in N} \mathcal{R}_{i} \subseteq \mathcal{R}^{n}$ is intertwined if for any agent $i, \mathcal{R}_{i}$ is intertwined.

Proving the equivalence between our elementary conditions and that of strategy proofness is a technical result. But remark that although easy to define, strategy proofness of a rule may be very hard to check. This difficulty vanishes when working on intertwinned domains, because then strategy proofness becomes equivalent to reshuffling invariance and monotonicity, which are both easy to check.

Our next application is, again, to prove an equivalence result between two normatively attractive requirements on social choice functions. We have already noted, in the preceding section, that all strategy proof social choice functions on (onedimensional) single peaked domains are also group strategy proof. And the same holds for other functions on that domain, and on a variety of other domains. But not always! Since we know that one of the two conditions implies the other, but not viceversa, we can ask: what is the common characteristic that separates those domains where the equivalence holds from those where the weaker condition does not imply the stronger one? In Barberà, Berga, and Moreno (2010) we have identified a condition that is sufficient (and in essence almost necessary) to imply this equivalence.

Given a preference profile $R_{N} \in \times_{i \in N} \mathcal{R}_{i}$ and a pair of alternatives $x, z \in A$, denote by $S\left(R_{N} ; z, x\right) \equiv\left\{i \in N: z P_{i} x\right\}, L\left(R_{i}, x\right)=\left\{y \in A: x R_{i} y\right\}$, and $\bar{L}\left(R_{j}, z\right)=\{y \in A:$ $\left.z P_{j} y\right\}$. We define a binary relation $\succsim\left(R_{N} ; z, x\right)$ on $S\left(R_{N} ; z, x\right)$ as follows: $i \succsim\left(R_{N} ; z, x\right) j$ if $L\left(R_{i}, x\right) \subseteq \bar{L}\left(R_{j}, z\right)$.

A preference profile $R_{N} \in \times_{i \in N} \mathcal{R}_{i} \subseteq \mathcal{R}^{n}$ satisfies sequential inclusion if for any pair $z, x \in A$ the binary relation $\succsim\left(R_{N} ; z, x\right)$ on $S\left(R_{N} ; z, x\right)$ is complete and acyclic.

For preferences $R_{i}, R_{i}^{\prime} \in \mathcal{R}_{i}$ and alternative $x \in A, R_{i}^{\prime}$ is a strict monotonic transformation of $R_{i}$ at $x$ if $R_{i}^{\prime}$ is such that for all $y \in A \backslash\{x\}$ such that $x R_{i} y, x P_{i}^{\prime} y$.

Let $R_{N}^{\prime}, R_{N} \in \times_{i \in N} \mathcal{R}_{i}$ be two preference profiles and let $x \in A$. We say that $R_{N}^{\prime}$ is a strict monotonic transformation of $R_{N}$ at alternative $x$ if for any $i \in N$, either $R_{i}^{\prime}=R_{i}$ or else $R_{i}^{\prime}$ is a strict monotonic transformation of $R_{i}$ at $x$.

A domain $\times_{i \in N} \mathcal{R}_{i}$ satisfies indirect sequential inclusion if, for all profiles $R_{N} \in \times_{i \in N} \mathcal{R}_{i}$, either (a) the profile $R_{N}$ satisfies sequential inclusion; or else (b) for each pair $z, x \in A$ there exists $R_{N}^{\prime} \in \times_{i \in N} \mathcal{R}_{i}$ where $R_{N \backslash S\left(R_{N} ; z, x\right)}^{\prime}=R_{N \backslash S\left(R_{N} ; z, x\right)}$, such that (1) $R_{N}^{\prime}$ is a strict monotonic transformation of $R_{N}$ at $x,(2)$ for any $i \in S\left(R_{N} ; z, x\right), z P_{i}^{\prime} x$, and $(3) \succsim\left(R_{N}^{\prime} ; z, x\right)$ is complete and acyclic.

In domains satisfying indirect sequential inclusion, strategy proof rules must also be group strategy proof. ${ }^{8}$ Moreover, given any domain violating the condition, it is possible to construct a social choice function that on that domain and for which the implication does not hold..$^{9}$

\footnotetext{
${ }^{8}$ See Le Breton and Zaporovhets (2009) for a related domain condition.

${ }^{9}$ For details about this "almost necessity" result, see Barberà, Berga, and Moreno (2010). The argument is not quite a necessity implication, and in that sense is reminiscent of previous definitions of "necessity" in social choice, like the one used in Sen and Pattanaik (1969).
} 
While still a technical result, this one has an important implication, because it identifies conditions under which even the coalition of all agents will not be able to jointly improve upon the outcome prescribed by the social choice function, and thus strategy proofness will be compatible with a weak notion of Pareto efficiency. Given the wide spread feeling that providing good incentives leads to inefficiency, the result helps to identify situations in which this conflict between two normatively attractive conditions need not arise.

An additional result in the same vein, but involving a different form of connectedness, applies in the case where alternatives consist of vectors of private consequences for selfish individuals. Rationing, matching or auctions are examples of economic problems that fit this general description and may be solved through the use of centralized mechanisms. The revelation principle ${ }^{10}$ allows us to study the conditions under which these mechanisms will have good incentive properties, by studying their associated direct mechanisms, that is, the social choice functions which relate the preferences of agents directly with the mechanisms' outcome. Individual and group strategy proofness are desirable properties for such direct mechanisms. One can observe that both conditions, individual and group strategy proofness, hold for the direct versions of mechanisms that solve matching, rationing, and allocation problems in contexts that are apparently very different from each other. In Barberà, Berga, and Moreno (2016) we have identified the common characteristics of these environments, proving that they all share some essential traits that can be expressed as a connectedness condition on domains.

The strict upper contour set of $R_{i}$ at $a_{i} \in B_{i}$ is $\bar{U}\left(R_{i}, a_{i}\right)=\left\{b_{i} \in B_{i}: b_{i} P_{i} a_{i}\right\}$.

A set of individual preferences $\mathcal{R}_{i}$ is rich if for any $R_{i}, \widetilde{R}_{i} \in \mathcal{R}_{i}, a_{i}, b_{i} \in B_{i}$ such that $b_{i} P_{i} a_{i}$, there exists $R_{i}^{\prime} \in \mathcal{R}_{i}$ such that $\bar{U}\left(R_{i}^{\prime}, b_{i}\right) \subseteq \bar{U}\left(R_{i}, b_{i}\right) \cap \bar{U}\left(\widetilde{R}_{i}, b_{i}\right), \bar{L}\left(\widetilde{R}_{i}, b_{i}\right) \subseteq \bar{L}\left(R_{i}^{\prime}, b_{i}\right)$, $\bar{U}\left(R_{i}, a_{i}\right)=\bar{U}\left(R_{i}^{\prime}, a_{i}\right)$ and $\bar{L}\left(R_{i}, a_{i}\right)=\bar{L}\left(R_{i}^{\prime}, a_{i}\right)$. A domain of preferences $\times_{i \in N} \mathcal{R}_{i}$ is rich if for any $i \in N, \mathcal{R}_{i}$ is rich.

Again, in that context, one can prove that the equivalence between individual and group strategy proofness is precipitated by the richness of the domains of definition.

We have laid down a catalog of similar conditions that can be used to solve a variety of questions regarding the role of domains in precipitating the compatibility or the equivalence of different normative requirements. These conditions share common characteristics. The most essential one is that they demand the possibility to connect pairs of preference profiles through sequences of changes, each one involving one agent's preference, and that the changes that are considered admissible, at each step, depend on a reference alternative. This is obvious for some of the conditions, like strong path connectedness, where at each step there is a reference alternative that becomes top for the changing agent. Likewise, the one requiring domains to be linked also involves changes from one preference to another that depend on a reference alternative, which is used to impose a monotonicity condition on the changing preferences. In other cases, the reformulation of the original statement into the common terms is not so direct, but still possible.

\footnotetext{
${ }^{10}$ See for example Gibbard (1973), Myerson (1979), Dasgupta, Hammond, and Maskin (1979) and Harris and Townsend (1981).
} 


\section{Final remarks}

We have tried to show by example how one of the many research lines that Arrow opened for all of us to follow has maintained its vitality till our days. The account is certainly biased and incomplete, but the main point regarding Arrow's pioneering role in this and so many other fields would only be strengthened by further additions.

Let us just remark that the importance of domain conditions is not limited to the study of social choice, where domains involve profiles of preference relations. It is also a relevant concern for other related classes of problems, like those considered by extensions of the Condorcet jury theorem to study communication and debate in committees, or those raised by the literature on judgement aggregation. In particular, defining domains is an important ingredient in mechanism design, where the characteristics of agents are described in a comprehensive way, through the language of types, that include not only what agents prefer, but also what they know, what they believe, how they process the information they get by themselves or through communication with others. There, the domains on which a mechanism is defined are represented by type profiles, and similar considerations than the ones mentioned in this essay, regarding the role of domain conditions, could be added to the ones we made here. ${ }^{11}$

Ackowledgements. We are thankful to the Journal Editor, Mark Fleurbaey, and to two extremely helpful referees, for comments and suggestions. We are also grateful to the editors of this special issue for allowing us to participate in this homage to Kenneth Arrow. S. Barberà acknowledges financial support through grants ECO2014-53052-P and SGR2014515, and Severo Ochoa Programme for Centres of Excellence in R\&D (SEV-2015-0563). D. Berga and B. Moreno acknowledge the financial support from the Spanish Ministry of Science, Industry and Competitiveness through grants ECO2016-76255-P and ECO201786245-P, respectively, and thank the MOMA network.

\section{References}

Arrow, K.: Social Choice and Individual Values. 2nd edition New York: Wiley (1st edition 1951) (1963)

Arrow, K., Sen, A., and Suzumura, K., editors: Handbook of Social Choice and Welfare, Volume 1, North Holland-Elsevier (2002)

Arrow, K., Sen, A., and Suzumura, K., editors: Handbook of Social Choice and Welfare, Volume 2, North Holland-Elsevier (2010)

Aswal, N., Chatterji, S., Sen, A.: Dictatorial domains. Economic Theory 22: 45-62 (2003)

Austen-Smith, D., Banks, J.: Information aggregation, rationality, and the Condorcet jury theorem. The American Political Science Review 90: 34-45 (1996)

Austen-Smith, D., Feddersen, T.: Deliberation and Voting Rules. In Austen-Smith, D. and J. Duggan (eds) Social Choice and Strategic Decisions: Essays in Honor of Jeffrey S. Banks, Berlin: Springer (2005)

\footnotetext{
${ }^{11} \mathrm{~A}$ recent attempt in that direction may be found in Barberà, Berga, and Moreno $(2018,2019)$.
} 
Austen-Smith, D., Feddersen, T.: Deliberation, preference uncertainty and voting rules. American Political Science Review 100(2): 209-21 (2006)

Baigent N.: Topological Theories of Social Choice, chapter 18 in Arrow, Sen and Suzumura (eds) Handbook of Social Choice and welfare (vol 2) North Holland (2010)

Ballester, M.A., Haeringer, G.: A characterization of the single-peaked domain. Social Choice and Welfare 36: 305-322 (2011)

Barberà, S.: Pivotal Voters: A New Proof of Arrow's Theorem. Economics Letters 6: 13-16 (1980)

Barberà, S.: Strategy-Proofness and Pivotal Voters: A Direct Proof of the GibbardSatterthwaite Theorem. International Economic Review 24: 413-417 (1983)

Barberà, S.: Indifferences and Domain Restrictions. Analyse und Kritik 29: 146-162 (2007)

Barberà, S., Berga, D., Moreno, B.: Individual versus group strategy-proofness: when do they coincide?. Journal of Economic Theory 145, 1648-1674 (2010)

Barberà, S., Berga, D., Moreno, B.: Two necessary conditions for strategy-proofness: on what domains are they also sufficient?. Games and Economic Behavior 75: 490-509 (2012)

Barberà, S., Berga, D., Moreno, B.: Some new domain restrictions in social choice, and their consequences. V. Torra et al. editors Springer Verlag: MDAI 2013, LNAI 8234: 11-24 (2013)

Barberà, S., Berga, D., Moreno, B.: Group Strategy-Proofness in Private Good Economies. American Economic Review 106: 1073-1099 (2016)

Barberà, S., Berga, D., Moreno, B.: Restricted Environments and Incentive Compatibility in Interdependent Values Models. Available at SSRN: https://ssrn.com/abstract=3135163 or http://dx.doi.org/10.2139/ssrn.3135163, and BGSE WP 1024 (July 2018)

Barberà, S., Berga, D., Moreno, B.: Domains admitting ex post incentive compatible and respectful mechanisms: a characterization for the two alternatives case. In Trockel,W. (Ed.): Social Design - Essays in Memory of Leonid Hurwicz. Springer (2019)

Barberà S., Gul F., Stacchetti E.: Generalized Median Voter Schemes and Committees. Journal of Economic Theory 61: 262-289 (1993)

Barberà, S., Jackson, M.O., Neme, A.: Strategy-Proof Allotment Rules. Games and Economic Behavior 18: 1-21 (1997)

Barberà, S., Massó, J., Serizawa, S.: Strategy-Proof Voting on Compact Ranges. Games and Economic Behavior 25: 272-291 (1998)

Barberà, S., Massó, J., Neme, A.: Voting under Constraints. Journal of Economic Theory 76: 298-321 (1997)

Barberà, S., Massó, J., Neme, A.: Voting by Committees under Constraints. Journal of Economic Theory 122: 185-205 (2005)

Barberà, S., Moreno B.: Top monotonicity: a common root for single peakedness, single crossing and the median voter result. Games and Economic Behavior 73: 345-359 (2011)

Berga, D.: Strategy-proofness and single-plateaued preferences. Mathematical Social Sciences 35: 105-120 (1998)

Bergson A.: A reformulation of certain aspects of welfare economics. Quarterly Journal of Economics 52: 310-334 (1938) 
Black, D.: On the Rationale of Group Decision Making. The Journal of Political Economy 56: 23-34 (1948)

Black, D.: The Theory of Committees and Elections. Cambridge University Press (1958) Blau, J. H.: The Existence of Social Welfare Functions, Econometrica 25: 302-313 (1957) Border, K.: Social Welfare Functions for Economic Environments with and without the Pareto Principle, Journal of Economic Theory 29: 205-216 (1983)

Bordes, G., Campbell, D. E., Le Breton, M.: Arrow's theorem for economic domains and Edgeworth hyperboxes. International Economic Review 36: 441-454 (1995)

Bordes, G. and Le Breton, M. Arrovian theorems with private alternatives domains and selfish individuals. Journal of Economic Theory 47: 257-281 (1989)

Bordes,G., Le Breton, M.: Arrovian theorems for economic domains: assignments, matchings, and pairings. Social Choice and Welfare 7: 193-208 (1990)

Chattherji, S.,Massó, J.: On strategy proofness and the salience of single peakedness. International Economic Review 59: 163-189 (2018)

Chatterji, S. Sanver, R., Sen, A.: On domains that admit well-behaved strategy-proof social choice functions. Journal of Economic Theory 148: 1050-1073 (2013)

Chichilnisky, G.: Social Choice and the Topology of Spaces of Preferences. Advances in Mathematics 37: 165-176 (1980)

Chichilnisky, G.: Social Aggregation Rules and Continuity. Quarterly Journal of Economics 97: 337-352 (1982)

Chichilnisky, G., Heal G.: Necessary and Sufficient Conditions for a Resolution of the Social Choice Paradox. Journal of Economic Theory 31: 68-87 (1983)

Dasgupta, P., Hammond P., Maskin E.: The implementation of Social Choice Rules: Some Results on Incentive Compatibility. Review of Economic Studies 46: 185-216 (1979)

Davis O.A, DE Groot,M., Hinich, M.: Social preference orderings and majority rule. Econometrica 40: 147-157 (1972)

Demange, G.: Single-peaked orders on a tree. Mathematical Social Science 3: 389-396 (1982)

Ehlers, L., Storcken, T.: Arrow's Possibility Theorem for one-dimensional single-peaked preferences. Games and Economic Behavior 64: 533-547 (2008)

Fishburn, P.C.: The Theory of Social Choice. Princeton University Press (1973)

Fishburn, P.C.: Acyclic Sets of Linear Orders. Social Choice and Welfare 14: 113-124 (1997)

Fishburn, P.C.: Acyclic sets of linear orders. A progress report. Social Choice and Welfare 19: 431-447 (2002)

Gaertner, W.: Domain Conditions in Social Choice Theory, Cambridge University Press (2001)

Gaertner, W.: Domain restrictions, Chapter 3 in Arrow, K., Sen,A., and Suzumura,K. (editors), Handbook of Social Choice and Welfare, Volume 1: 131-170, North Holland (2002)

Gaertner, W.: De jure naturae et gentium: Samuel von Pufendorf's contribution to social choice theory and economics. Social Choice and Welfare, 25: 231-241 (2005)

Gaertner, W.: Kenneth Arrow's impossibility theorem stretching to other fields, Public Choice, 179: 125-131 (2019) 
Gans, J.S., Smart, M.: Majority voting with single-crossing preferences. Journal of Public Economics 59: 219-237 (1996)

Gibbard, A.: Manipulation of Voting Schemes: A General Result. Econometrica 41: 587-601 (1973)

Harris, M., Townsend, R.: Resource Allocation Under Asymmetric Information. Econometrica 49: 33-64 (1981)

Igersheim, H.: The death of welfare economics. History of a controversy. The Center for the History of Political Economy Working Paper, No. 2017-03 (2017)

Kalai, E., Muller, E., Satterthwaite, M.: Social Welfare Functions when Preferences are Convex and Continuous: Impossibility Results. Public Choice 34: 87-97 (1979)

Kramer, G. H.: On a class of equilibrium conditions for majority rules. Econometrica 41: 285-297 (1973)

Le Breton, M., Sen, A.: Strategyproofness and Decomposability: Weak Orderings. G.R.E.Q.A.M. 95a38, Université Aix-Marseille III (1995)

Le Breton, M., Sen, A.: Separable Preferences, Strategyproofness and Decomposability. Econometrica 67: 605-628 (1999)

Le Breton, M., Weymark, J.W.: Arrovian Social Choice Theory on Economic Domains, Chapter 17 in Handbook of Social Choice and Welfare, Arrow, K., Sen, A. and K. Suzumura (Eds), Elsevier (2010)

Le Breton, M., Zaporozhets, V.: On the equivalence of coalitional and individual strategyproofness properties. Social Choice and Welfare 33: 287-309 (2009)

List, C., Polak, B.: Introduction to Judgment Aggregation. Journal of Economic Theory 145: 441-466 (2010)

List, C.: The theory of judgment aggregation: an introductory review, Synthese 187: 179-207 (2012)

Maskin, E.: Social welfare functions for economics. Unpublished manuscript, Darwin College, Cambridge University and Department of Economics, Harvard University (1976)

Maskin, E.: Fonctions de Préférence Collective Définies sur des Domaines de Préférences Individuelles Soumis à des Contraintes, Cahiers du Séminaire d'Econométrie 20: 153-182 (1979)

Massó, J., Moreno de Barreda, I.: On strategy-proofness and symmetric single peakedness. Games and Economic Behavior 72: 467-484 (2011)

McKelvey, R. D.: Intransitivities in multidimensional voting models and some implications for agenda control. Journal of Economic Theory 12: 472-482 (1976)

McKelvey, R.D.: Social Choice. The division of Humanities and Social Sciences, California Institute of Technology (1996)

Moulin, H.: On Strategy-proofness and Single Peakedness. Public Choice 35: 437-455 (1980)

Moulin, H.: Generalized Condorcet-Winners for Single Peaked and Single Plateau Preferences. Social Choice and Welfare 1: 127-147 (1984)

Muller, E.: Graphs and Anonymous Social Welfare Functions, International Economic Review 23: 609-622 (1982)

Myerson, R.: Incentive Compatibility and the Bargaining Problem. Econometrica 47: 61-73 (1979) 
Nehring, K, Puppe, C.: The structure of strategy-proof social choice - Part I: general characterization and possibility results on median spaces. Journal of Economic Theory 135: 269-305 (2007)

Penn, E.M., Patty, J.W., Gailmard, S.: Manipulation and Single-Peakedness: A General Result. American Journal of Political Science 55: 436-449 (2011)

Plott, C.R.: A notion of equilibrium and its possibility under majority rule. American Economic Review 57: 787-806 (1967)

Redekop, J.: Social Welfare Functions on Restricted Domains, Journal of Economic Theory 53: 396-427 (1991)

Roth, A, What have we learned from market design, Hahn Lecture, Economic Journal, 118: 285-310 (2008)

Samuelson P.A.: Foundations of Economic Analysis. Harvard University Press, Cambridge, MA (1947)

Saporiti, A., Tohmé. F.: Single-crossing, strategic voting and the median choice rule. Social Choice and Welfare 26: 363-383 (2006)

Satterthwaite, M.: Strategy-proofness and Arrow's conditions: Existence and correspondence theorems for voting procedures and social welfare functions. Journal of Economic Theory 10: 187-217 (1975)

Schmitz, N.: A Further Note on Arrow's Impossibility Theorem. Journal of Mathematical Economics 4: 189-196 (1977)

Schofield, N.: Instability of Simple Dynamic Games. The Review of Economic Studies 45, 575-594 (1978)

Sen, A.: A possibilty theorem on majority decisions. Econometrica 34: 491-499 (1966)

Sen A., Pattanaik P.K.: Necessary and sufficient conditions for rational choice under majority decision. Journal of Economic Theory 1: 178-202 (1969)

Sprumont, Y.: The Division Problem with Single-Peaked Preferences: A Characterization of the Uniform Allocation Rule. Econometrica 59: 509-519 (1991) 